\title{
THE EFFECT OF PERCEIVED TRANSFORMATIONAL LEADERSHIP STYLE ON EMPLOYEE ENGAGEMENT: THE MEDIATING EFFECT OF LEADER'S EMOTIONAL INTELLIGENCE
}

\author{
Marwan MILHEM*, Habsah MUDA**, Khalil AHMED*** \\ *Universiti Sultan Zainal Abidin, Faculty of Economics and Management Sciences, Terengganu, MALAYSIA \\ e-mail: mmarwanmilhem@gmail.com \\ **Universiti Sultan Zainal Abidin, Faculty of Economics and Management Sciences, \\ Department of Management Sciences, Terengganu, MALAYSIA \\ e-mail: habsah_muda@unisza.edu.my \\ ***International Islamic University Malaysia, Institute of Islamic Banking and Finance, \\ Selangor, MALAYSIA \\ e-mail: khaleel8344814@yahoo.com
}

\begin{abstract}
Employee engagement has been at the center of attention for human resource personnel and researchers who stated that employee engagement can be a key driver of an organization's business success. Many researchers have found in recent researches that a transformational leadership style has a beneficial connection with employee engagement. Thus, this study aims to find the causal effect of perceived transformational leadership on employee engagement through the mediating role of leader's emotional intelligence. We analyze the data from a sample of 338 full-time employees working in the ICT sector in Palestine using confirmatory factor analysis (CFA) and structural equation modelling (SEM) to test the hypothesis. Later, the bootstrapping analysis was conducted to confirm the mediation test in this study. The finding shows that transformational leadership style has a significant effect on employee engagement for the mediating role of emotional intelligence. The effect of transformational leadership on employee engagement was found to be completely mediated by leader's emotional intelligence at work. Theoretically, this empirical study contributed to the new knowledge on the unique mediating effects of leader's emotional intelligence in the relationship between transformational leadership and employee engagement in the Middle Eastern Region. Practically, this study has managerial implication in the area of organizational management, leadership and human resource management.
\end{abstract}

Keywords: employee engagement (EE), emotional intelligence (EI), transformational leadership, exploratory factor analysis (EFA), confirmatory factor analysis (CFA), information \& communication technology (ICT), structural equation modelling (SEM).

JEL: M1, M10, M12.

\section{Introduction}

Business employee engagement, staff engagement and work engagement and worker engagement are the labels used to describe features, evaluate the wellbeing of employees at work, and measure the influences on the total organizational outcomes. Recently, the effect of employee engagement has become an important issue for individual, organizational, and macroeconomic levels. Organizations seek to hire employees who can adapt themselves to the changes and are proactive, energetic, and creative while facing problems and challenges. Contemporary challenges in the workplace causes decision- makers to think about investing in human capital, thus, the concept of employee engagement has become common as a method to increase the labor productivity, compete highly in the international economy, and achieve organizational objectives.

According to a recent study of 1000 global organizations by Hewitt (2017), employee engagement peaked in 2015 at $65 \%$, but its gains were wiped away with a two-point drop in 2016 at $63 \%$ and in 2018 according to a recent report by Aon Hewitt, employee engagement matched the previous peak at $65 \%$. 
Agreeing to the study, there are some factors that affect the decline in employee engagement, such as populism (resentment towards foreign workers and open borders) which is caused by the prevailing post Arab Spring in the Middle East and have a major impact on employee engagement because of the new restrictions and pressure put on employees especially foreigners. According to the same study in Asia Pacific, the overall engagement scores of employees in 2015 went from $65 \%$ down to $62 \%$ in 2016 . Aon's analysis reported that regional and country-specific political, cultural, and economic differences drive regional variations in engagement.

The research reported from over 717 HR profession related members in the employee engagement worldwide; it was found that only $39 \%$ of average organizations say their senior leaders prioritize engagement. Similarly, whereas only $28 \%$ of all respondents said their managers were highly skilled at fostering engaged individuals and teams. Thus, leadership talents are the main differentiators between highly engaged organizations and average organizations (HR. Com, 2018).

A large and growing body of literature has investigated and concluded that leadership has a significant role in affecting employee engagement (Wallace and Trinka, 2009; Shuck and Herd, 2012). The recent Global Employee Engagement Index (2018), is a global survey conducted by Effectory International in 56 different countries worldwide and includes over 18,000 respondents.

The finding showed that global levels of employee engagement and commitment are still less than 30\% and continue to rise very slowly, with an increase of just $4 \%$ over the last four years, which means that organizations have a wealth of untapped talent being wasted, and in the worst case scenario, draining company resources and space for improvement. Moreover, the outcomes of this report showed that outstanding leader and inspiring immediate managers are the main factor driving employee engagement.

In a case study by Freedman (2013), emotional intelligence was shown as predicting $47 \%$ of the difference in the range of the manager's performance scores. Also, it strongly correlates the higher organizational engagement. Managers' emotional intelli- gence was shown to predict $76 \%$ of variation in engagement, though, within the course of the study, employee turnover was reduced to $63 \%$. For many decades' leadership was considered a significant subject academically and organizationally.

According to Bass and Riggio (2006), the transformational leader is seen as the person who has certain personal or leadership characteristics, like inspirational motivation, intellectual stimulation, idealized impact (charisma), and individual consideration that assists himlher to go beyond the simple transactions or just reward exchange relationships with subordinates and share values, needs, and vision of the future.

A recent study by Liu and Cho (2018) showed that managers with a high level of emotional intelligence are considered as asset of their companies, and leaders' EI could even interact with the employees to help them get engaged in the work.

Carasco-Saul, et al. (2015) reviewed literatures and used multiple databases encompassing 39 smaller database subsets (e.g., ABI/INFORM Complete, Proudest Education Journals, PsycINFO, and PsycARTICLES) to extract as many relevant articles as possible for their study to examine the relationship between leadership and employee engagement.

Through this process, 20 articles out of 81 were chosen for further review where 16 of them were empirical and 4 were conceptual. However, according to the authors, the relationship between leadership and employee engagement had not been widely investigated, though, many organizations invested important resources in retraining, developing, and engaging employees.

In the study of Ababneh (2015), they are found the absence of critical examination of the moderating and mediating influence of organizational culture and job characteristics which presents the direct relationship between the antecedents of engagement (leadership and personality) and employee engagement.

Though, a lot of writing has been done on employee engagement, Shuck and Wollard (2010) found that serious academic researchers had not been published sufficiently. This gap has resulted in a disjointed 
approach to understand and develop strategies around employee engagement within organizations.

This study tried to define and situate the concept within the HRD field through a historical lens by using an integrated literature review and explored the development of employee engagement by systematically reviewing and organizing literatures across various disciplines and fields of study.

Several researchers have suggested various processes through which transformational leaders influence employees to be engaged. An underestimated and interesting pathway is the leaders' ability to design meaningful and motivating work (Serrano and Reichard, 2011). Bakker, et al. (2011) noted the importance of this concept and argued that this influence can result from helping employees construe meaningful in their works. Lack of empirical support was found to confirm this suggested pathway. Bakker (2011) suggested that the current study regarding leadership does not completely explain "how leaders influence their followers" and what techniques explain this influence.

Zhu, et al. (2009) showed that there is a direct relationship between transformational leadership and employee engagement; however, they argued that others have neglected the constructs that might indirectly affect employee engagement which must be tested and researched. Shuck and Herd (2012) found that leadership behavior can significantly influence these factors.

However, it is not yet understood clearly about the process of this influence, or leadership behaviors that affect employee engagement. Moreover, there is still a gap in understanding which leadership behaviors impact engagement-encouraging cultures or exactly how leader behaviors cause improvements in engagement.

Arunima, et al. (2014) investigated the relationships between perceptions of leaders' emotional intelligence and transformational and transactional leadership styles as assessed by doctors. The findings of this study demonstrated a moderate correlation $(r=0.310)$ between emotional intelligence and transformational leadership style. Many researchers argued that there are still lots of issues in a leadership style that must be determined for better understanding of the influence of emotional intelligence on behavior (Ybarra, et al., 2014).

Accordingly, Thor (2013) found a strong positive link between emotional intelligence and employee engagement exists; emotional intelligence alone does not fully explain the variability in work engagement. Later, Ochalski (2016) showed that a leader's emotional intelligence did not seriously moderate the relationship between transformational leadership, employee engagement in USA culture but might moderate or mediate in other cultures.

Researches on emotional intelligence and employee engagement are growing. Recent studies conducted by Ravichandran, et al. (2011), Thor (2013), and Webb (2013) confirmed an important and beneficial link between emotional intelligence and employee engagement. These findings suggest that emotional intelligence on its own may not be able to explain the variability in employee engagement properly. Thus, assessing employee engagement factors other than emotional intelligence may have to be considered.

However, empirical research, linking to the mediating role of leaders' emotional intelligence to the specific attributes of the employee engagement under investigation was lacking. With this gap in knowledge, we conceptualize that leader's emotional intelligence can act as a possible mediator between transformational leadership and employee engagement relationship (Fig. 1). Therefore, this study presents the following hypothesis:

Does a leader's emotional intelligence mediate the effect of transformational leadership on employee engagement?

H1. Leader's emotional intelligence does mediate the effect of transformational leadership on employee engagement. 


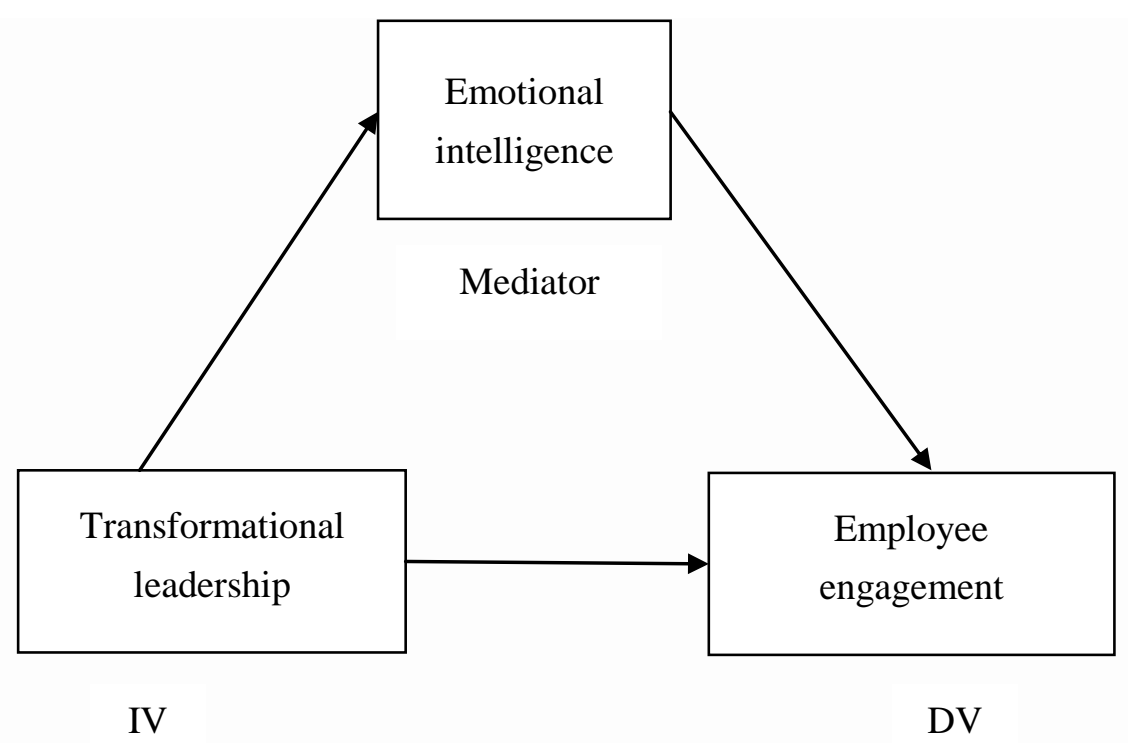

Figure 1. The Schematic Diagram of a Model under Study (Source: Authors' own research)

\section{$2 \quad$ Methodology}

This study applies the quantitative research approach involves measuring data on constructs (transformational leadership, emotional intelligence and employee engagement), using quantitative scales and quantitative data analysis. Prior to field studies, we perform an exploratory factor analysis (EFA) based on the 100 samples from the pilot study to confirm the dimensions of the items and check an internal reliability for each construct under study.

\subsection{Sampling and data collection}

In the field study, a total of 450 questionnaires were distributed based on simple random sampling in the targeted full time employees in the ICT sector in Palestine. The questionnaires were provided with instructions to complete all the points properly. Invitation letters were also provided which described the aims and objectives of the study, potential contribution, data collection, potential use of the research, and how confidentiality would be maintained to secure privacy. The numbers of questionnaires returned by the respondents were 348, which represented a return rate of $77 \%$. 10 of the survey responses were incomplete. Thus, 338 samples were screened and treated for further analysis using IBM SPSS AMOS 21.

\subsection{Measurement of construct}

We employ a 10-point rating scales to measure the level of employee's perception towards the itemized statement in the questionnaire involving transformational leadership, emotional intelligence and employee engagement to meet the requirement for data assumption using parametric analysis.

\subsection{Transformational leadership (TL)}

The leadership style scale assessed via the multifactor leadership questionnaire (MLQ-5X version; Bass and Avolio, 2004) was used. The MLQ is a broadly used measure consisting of 45 items. However, only 16 items evaluated followers' own perceptions. This construct has four sub-constructs namely idealized influence behavior (TLB) (measured using 3 items), inspirational motivation (TLM) (measured using 3 items), intellectual stimulation (TLS) (measured using 6 items), and individual consideration (TLC) (measured using 4 items). The participants evaluated their immediate supervisor's behavior for each statement under the transformational leadership, by using a 10-point interval scale. 


\subsection{Emotional intelligence (EI)}

Emotional intelligence was measured by using the Genos Emotional Intelligence Inventory-concise (rater) scale which was designed to measure the overall emotional intelligence score as well as the emotional intelligence subscales based on 39 items (Gignac, 2010b).

This construct has four sub-constructs namely emotional self-awareness (EIA) (measured using 3 items), emotional reasoning (EIR) (measured using 4 items), emotional management of others (EIO) (measured using 7 items), and emotional selfmanagement (EISM) (measured using 9 items).

\subsection{Employee engagement (EE)}

Employee engagement was measured by using the Utrecht Work Engagement Scale (UWES) developed by Schaufeli and Bakker (2003). This concept basically is a second order measured by using three subconstructs namely dedication ED (measured using 7 items), vigor EV (measured using 3 items), and absorption EA (measured using 3 items).

\subsection{Demographic profile}

Employees were asked to provide information about their age group, gender, company size, current position, time to hold current position, and time to work with current manager at the time of data collection.

\section{$3 \quad$ Finding and discussion}

\subsection{The assessment of normality for data set}

In the normality assessment of this study, we observed the measure of skewness, which reflected the normal distribution for every item under each construct of transformational leadership (TL), emotional intelligence (EI), and employee engagement (EE). The values of skewness, which fell within the range of -1.3 and 1.6, indicating that the distribution of data in this study was normal.

\subsection{Confirmatory factor analysis (CFA)}

Using confirmatory factor analysis (CFA) we examined the fitness indices for the model. These indices showed how well the proposed model captured the covariance among items in the measurement model of transformational leadership, emotional intelligence, and employee engagement. Once the CFA report is completed and all values meet the required thresholds for validity and reliability, we conclude that the measurement models for all latent constructs involved in the model have been validated (Awang, 2015).

Then, the next step, we assemble these constructs into the structural model to execute structural equation modelling (SEM). We arrange the constructs from left to right, beginning with the exogenous constructs (far left) followed by the mediator constructs (in the middle), and the endogenous construct (far right) (Awang, 2015).

Then, based on the direction of the hypothesis, we link the exogenous construct to its respective endogenous construct by using the single headed arrow (Fig. 2). Based on the structural model in Fig. 2, we had set the AMOS to compute the values for the standardized regression weights (standardized estimate) and the regression weights (unstandardized estimate) for the model.

The fit indices showed that the higher-order factor of the model fitted the data adequately. The fitness index for the measurement model was extracted and the result showed that the higher-order factor of the model fitted the data adequately:

$$
\begin{aligned}
& \text { CMIN/DF }=2.31, \\
& \text { RMSEA }=0.059, \\
& \text { CFI }=0.902, \\
& \text { IFI }=0.903 .
\end{aligned}
$$

Under the "close fit" hypothesis (i.e., the RMSEA is no greater than 0.05 in the population), the probability of getting a sample RMSEA as large as 0.080 was 0.059 .

The entire factor loading of the remaining items, as shown in Fig. 2, had exceeded the required value of 0.6. Thus, we could assume that the unidimensionality of the measurement model had been achieved. Hence, we satisfied with the obtained fit- 
ness index and the CFA procedure was completed at this stage before proceeding the structural modelling.

The requirement for composite reliability (CR), and average variance extracted (AVE) were achieved for all items factoring as:
- transformational leadership, $\mathrm{CR}=0.92$ and AVE was 0.76 ,

- emotional intelligence, $\mathrm{CR}=0.94$, and AVE was 0.80 , and

- employee engagement, $\mathrm{CR}=0.88$, and AVE was 0.72 .

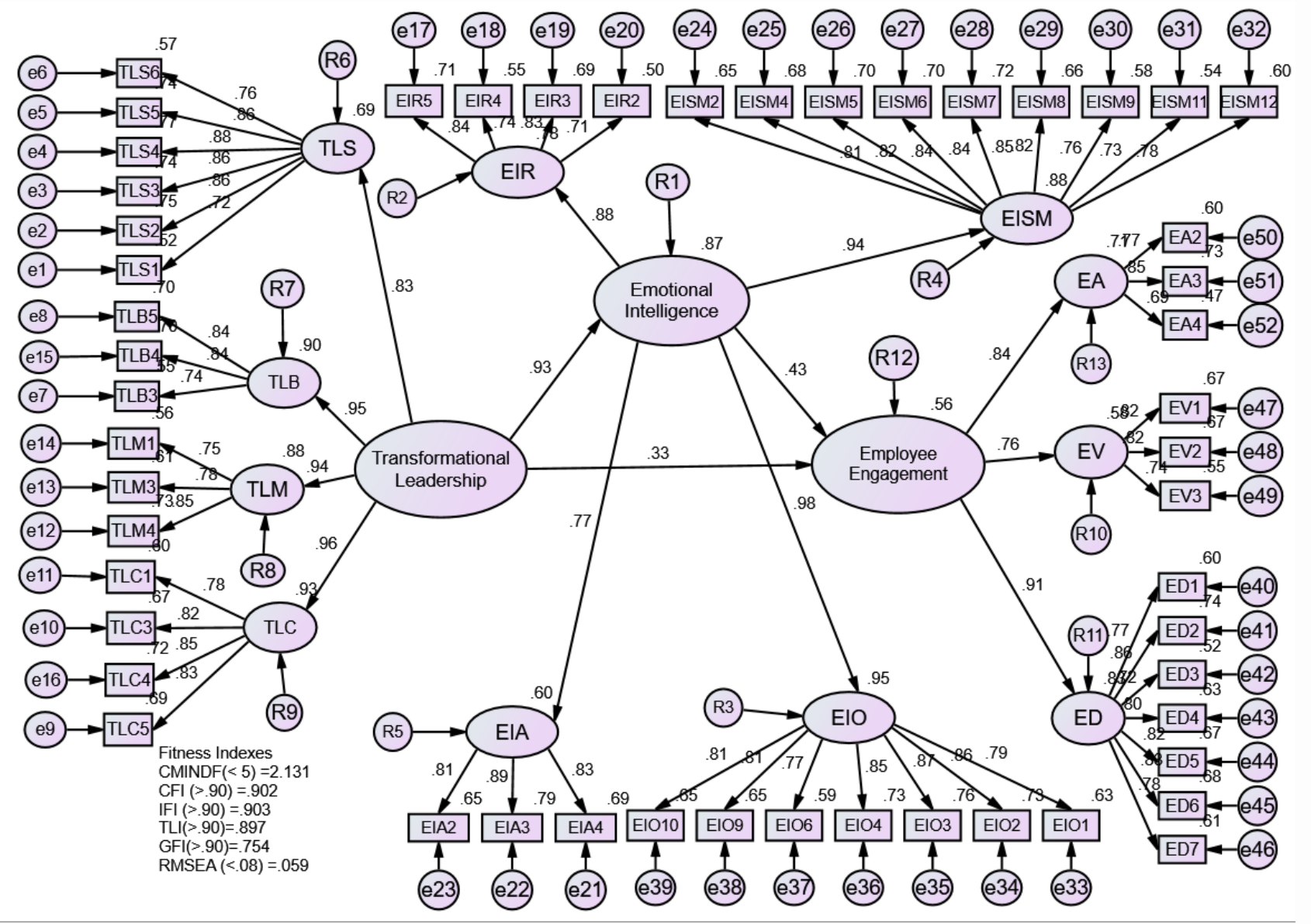

Figure 2. AMOS output showing the position of EI (mediator) between TL and EE (Source: Authors' own research )

\subsection{Hypothesis testing: analyzing mediating variables of emotional intelligence on the effect of transformational leadership on employee engagement}

In this study, we hypothesize emotional intelligence as a construct that mediates the effect from an exogenous construct of transformational leadership to its endogenous construct of employee engagement. The assumption is that if the direct effect of TL to EE does not exist in the structural model, but the effect exists indirectly through another construct of EI; then in this study EI is a mediating constructs.
Fig. 2 shows the position of EI between TL and EE.

The result showed that the probability of getting a critical ratio of 12.379 in absolute value was less than 0.001. In other words, the regression weight for TL when predicting EI significantly differed from zero at 0.001 levels.

Besides, the probability of obtaining a critical ratio of 1.851 in absolute value was 0.064 . In other words, the regression weight for TL when predicting EE did not significantly differ from zero at 0.05 levels (twotails). 
The probability of obtaining a critical ratio of 2.363 in absolute value was 0.018 . In other words, the regression weight for EI when predicting EE significantly differed from zero at 0.001 levels (two-tails). Based on the analysis, the above research hypothesis was supported.

This study concludes that EI has significantly mediated the relationship between TL and EE. As illustrated before, the type of mediation is called a "complete mediation" since the direct effect of the TL on EE was no longer significant after EI entered the model. The beta coefficient for TL was reduced from 0.575 to 0.241 . Therefore, in this study, TL had a significant indirect effect on EE through the mediator variable, namely EI.

\subsection{The procedure for testing mediation of emotional intelligence (EI)}

The procedure for testing mediation of emotional intelligence:

1) The indirect effect $=0.93 \times 0.43=0.40$;

2) The direct effect $=-0.33$;

3) Both indirect paths (TL to EI and EI to EE) are significant;
4) Since indirect effect $>$ direct effect, the mediation occurs;

5) Type of mediation is complete mediation since the direct effect is not significant after EI (mediator) enters the model.

In testing the hypothesis for the mediation effect of EI in linking TL with EE, the hypothesis was supported. Consequently, this study revealed that EI has significantly mediated the relationship between (TL) and (EE). Thus, these findings have answered the research question: Does a leader's emotional intelligence mediate the effect of transformational leadership on employee engagement?

To discover the type of mediation, these findings also provided a calculation for the effect size of mediation. In calculating the effect size of a mediator in the mediating effect, it was found that the effect size of EI on EE was in the small range.

In this study, the effect size is the amounts of variance explained in the mediation model contribute by every single path. Again, these findings closed the gaps found in the previous researches by linking EI as a mediator in the relationship between TL and EE. This study has not only revealed the type of mediation, but also the effect size of EI on EE.

Table 1. Testing EI as a mediator in the relationship between TL and EE

(Source: Authors' own research)

\begin{tabular}{|c|c|c|l|}
\hline Relationship & Standardized $(\beta)$ & P-Value & \multicolumn{1}{|c|}{ Result } \\
\hline $\mathrm{TL} \rightarrow$ EI (a) & 0.94 & $* * *$ & Significant \\
\hline $\mathrm{EI} \rightarrow$ EE (b) & 0.43 & 0.18 & Significant \\
\hline $\mathrm{TL} \rightarrow$ EE (c) & 0.332 & 0.064 & Not Significant \\
\hline $\mathrm{a} * \mathrm{~b}$ & 0.40 & \\
\hline $\mathrm{a} * \mathrm{~b}>\mathrm{c}$ & Mediation occurs & \\
\hline
\end{tabular}

Based on the above test, the mediation test is supported, and the type of mediation is complete mediation since the direct effect is not significant.
However, we need to confirm the mediation effect outcomes with the outcomes of direct effect and indirect effect from Bootstrapping. The Bootstrapping result is shown in Table 2. 
Table 2. Bootstrapping result

(Source: Authors' own research)

\begin{tabular}{|c|c|c|}
\hline & $\begin{array}{c}\text { Indirect Effect } \\
\text { P-Value }\end{array}$ & $\begin{array}{c}\text { Direct Effect } \\
\text { P-Value }\end{array}$ \\
\hline Bootstrapping P-Value & 0.000 & 0.064 \\
\hline Result & Significant & Not Significant \\
\hline Type of Mediation & \multicolumn{2}{|c|}{ Complete Mediation } \\
\hline
\end{tabular}

\section{Conclusion}

This study focused the mechanism underlying the effects of transformational leadership style on employee engagement from the perspective of mediating part of leaders' emotional intelligence. The finding of this study indicate that leaders' emotional intelligence adds to the strengthening of the relationship between transformational leadership and employee engagement and there was effect role of the mediators when inter the model and the size of this effect was in small range.

These results address Ochalski (2016) conceptual claims that EI might mediate the relationship between transformational leadership and employee engagement in different cultural contexts. This study also supports Ghadi, et al. (2013) recommendation that other mediators may affect the relationship between TL and EE which need to study. In this regard, this research framework can be extended to further studies in another context other than ICT sector. While most engagement studies have heavily focused on linking employee engagement to organizational work conditions (e.g. Job resources and job demands), this study provides empirical evidence demonstrating that employee engagement reflects a two-way relationship.

The findings of this study contribute to the scholarly literature by providing important information regarding the relationships between leadership styles, leaders' emotional intelligence, and employee engagement, which have not been previously investigated in a single study. From a practitioner's standpoint, the findings from this study suggest that for leaders who show the transformational leadership behavior of emotional, inspirational, and motivation are more suitable for engaging employees at work place. In general, this research has many practical implications for the organizational development, HR professionals, leaders and employees themselves.

Specifically, these implications address issues related to managing performance, training and development, job design, recruitment and selection. The possible limitation this study only can be generalized to a population of employees working in the ICT sector in Palestine due to different culture in different context of studies.

Additionally, the finding originates from current research suggested that there is a need for future research based on the evaluations of the specific dimensions of emotional intelligence affecting employee engagement require additional investigation may be in the importance of emotional intelligence behaviors that consider both the leader and employees, rather than the emotional intelligence behaviors that describe the behaviors of only the leader.

Acknowledgement: The authors would like to thank all friends who assisted them to process this paper.

\section{$5 \quad$ References}

[1] Ababneh, O.M.A., 2015. Conceptualizing and measuring employee engagement, and examining the antecedents of leadership styles and personality attributes (Doctoral dissertation, Auckland University of Technology).

[2] Arunima, S., Ajeya, J., Sengupta, A.K., Mariamma, P., Tripathi, K.K., 2014. Comparison of transformational leadership and its different attributes of leadership with emotional intelligence 
in Indian healthcare: An empirical aspect. Advances in Management, 7(10), p.37.

[3] Awang, Z., 2015. SEM Made Simple: A Gentle Approach to Learning Structural Equation Modeling. Bandar Baru Bangi, MPWS Rich Resources.

[4] Bakker, A.B., Albrecht, S.L., Leiter, M.P., 2011. Key questions regarding work engagement. European journal of work and organizational psychology, 20(1), pp.4-28.

[5] Bass, B., Avolio, B., 2004. Manual for Multifactor Leadership Questionnaire: Sampler Set. Mind Garden Inc., Redwood City, CA.Bass, B.M. (1985).

[6] Bass, B.M., Riggio, R.E., 2006. Transformational leadership. Psychology Press.

[7] Carasco-Saul, M., Kim, W., Kim, T., 2015. Leadership and employee engagement: Proposing research agendas through a review of literature. Human Resource Development Review, 14(1), pp.38-63.

[8] Effectory International, 2016. Global Employee Engagement Index. https://www.effectory.com /knowledge/themes/employee-engagement-surv ey/.Amsterdam, Netherlands.

[9] Effectory, 2018. Global Employee Engagement Index. https://www.effectory.com/knowledge/ blog/discover-the-latest-global-employee-engag ement-and-commitment-insights/.Amsterdam, Netherlands

[10] Freedman, J., 2013. The Amadori Case: Supplying McDonalds - Organizational Engagement, Emotional Intelligence and Performance. Retrieved from: http://jmfreedman.com/2013/04/ the-amadori-case-supplying-mcdonalds-organiza tional.

[11] Gignac, G.E., 2010b. Genos emotional intelligence inventory technical manual. (2nd ed.). Retrieved from http://www.genosinternational .com/emotionalintelligence/ technical-manual.

[12] Hewitt, A., 2017. Trends in global employee engagement: Global anxiety erodes employee engagement gains. Talent, Rewards \& Performance.

[13] Liu, J., Cho, S., 2018. Interaction effect of display rules and emotional intelligence on hotel managers' and non-managers' work engage- ment. International Journal of Contemporary Hospitality Management, 30(3), pp.1903-1919.

[14] Ochalski, S., 2016. The moderating role of emotional intelligence on the relationship between transformational leadership and work engagement. International Leadership Journal, 8(2), pp.68-87.

[15] Ravichandran, K., Arasu, R., Kumar, S.A., 2011. The impact of emotional intelligence on employee work engagement behavior: An empirical study. International Journal of Business and Management, 6(11), p.157.

[16] Schaufeli, W.B., Bakker, A.B., 2003. Utrecht work engagement scale: Preliminary manual. Occupational Health Psychology Unit, Utrecht University, Utrecht, 26.

[17] Serrano, S.A., Reichard, R.J., 2011. Leadership strategies for an engaged workforce. Consulting Psychology Journal: Practice and Research, 63(3), p.176.

[18] Shuck, B., Herd, A.M., 2012. Employee engagement and leadership: Exploring the convergence of two frameworks and implications for leadership development in HRD. Human resource development review, 11(2), pp.156-181.

[19] Shuck, B., Wollard, K., 2010. Employee engagement and HRD: A seminal review of the foundations. Human resource development review, 9(1), pp.89-110.

[20] The State of Employee Engagement in 2018: Leverage leadership and culture to maximize engagement, from: https://www.hr.com/en/ resources/free_research_white_papers/the-stateof-employee-engagement-in-2018-mar2018_jeq fvgoq.html

[21] Thor, S. 2013. MOOD righting. Quality Progress, 46(5), pp.34-40.

[22] Wallace, L., Trinka, J., 2009. Leadership and employee engagement. Public Management, 91(5), pp.10-13.

[23] Webb, K.S., 2013. How managers' emotional intelligence impacts employees' satisfaction and commitment: A structural equation model. The IUP Journal of Organizational Behavior, 13(2), pp.7-24.

[24] Yasin Ghadi, M., Fernando, M., Caputi, P., 2013. Transformational leadership and work engagement: The mediating effect of meaning in 
work. Leadership \& Organization Development Journal, 34(6), pp.532-550.

[25] Ybarra, O., Kross, E., Sanchez-Burks, J., 2014. The "big idea" that is yet to be: Toward a more motivated, contextual, and dynamic model of emotional intelligence. Academy of Management Perspectives, 28(2), pp.93-107.
[26] Zhu, W., Avolio, B.J., Walumbwa, F.O., 2009. Moderating role of follower characteristics with transformational leadership and follower work engagement. Group \& Organization Management, 34(5), pp.590-619. 